\title{
On the Construction of Abstract Voronoi Diagrams*
}

\author{
K. Mehlhorn, ${ }^{1}$ St. Meiser, ${ }^{1}$ and C. Ó'Dúnlaing ${ }^{2}$ \\ ${ }^{1}$ FB Informatik, Universität des Saarlandes, \\ D-6600 Saarbrücken, Germany \\ ${ }^{2}$ Department of Mathematics, Trinity College Dublin, \\ Dublin, Ireland
}

\begin{abstract}
We show that the abstract Voronoi diagram of $n$ sites in the plane can be constructed in time $O(n \log n)$ by a randomized algorithm. This yields an alternative, but simpler, $O(n \log n)$ algorithm in many previously considered cases and the first $O(n \log n)$ algorithm in some cases, e.g., disjoint convex sites with the Euclidean distance function. Abstract Voronoi diagrams are given by a family of bisecting curves and were recently introduced by Klein [13]. Our algorithm is based on Clarkson and Shor's randomized incremental construction technique [7].
\end{abstract}

\section{Introduction}

The Voronoi diagram of a set of sites in the plane partitions the plane into regions, called Voronoi regions, one to a site. The Voronoi region of a site $s$ is the set of points in the plane for which $s$ is the closest site among all the sites. The Voronoi diagram has many applications in diverse fields, see [19] or [3] for a list of applications and a history of Voronoi diagrams. Different types of diagrams result from considering different notions of distance, e.g., Euclidean or $L_{p}$-norm or convex distance functions, and different sorts of sites, e.g., points, line segments, or circles; see also Section 4. For many types of diagrams efficient construction algorithms have been found; these are either based on the divide-and-conquer technique due to Shamos and Hoey [22], the sweepline technique due to Fortune [10], or geometric transforms due to Brown [5] and Edelsbrunner and Seidel [8].

A unifying approach to Voronoi diagrams was recently proposed by Klein [13]. He does not use the concept of distance as the basic notion but rather the concept

*This work was supported by the DFG, Me 620/6, and ESPRIT P3075 ALCOM. A preliminary version of this paper has been presented at STACS ' 90 , Rouen, France. 
of bisecting curves, i.e., he assumes for each pair $\{p, q\}$ of sites the existence of a bisecting curve $J(p, q)$ which divides the plane into a $p$-region and a $q$-region. The intersection of all $p$-regions for different $q$ 's is then the Voronoi region of site $p$. He also postulates that Voronoi regions are simply connected and partition the plane. He shows that abstract Voronoi diagrams already have many of the properties of concrete Voronoi diagrams, see Section 2. He also shows that the divide-andconquer technique can be used to construct abstract diagrams efficiently. More precisely, if the basic geometric operations on bisecting curves take time $O(1)$ and if any set $S$ of sites can be split in time $O(|S|)$ into about equal-sized subsets $L$ and $R$ such that the bisector between $L$ and $R$ (= the common boundary of regions in $L$ with regions in $R$ ) is acyclic, then the Voronoi diagrams of $L$ and $R$ can be merged in time $O(|S|)$ and hence the diagram of $n$ sites can be constructed in time $O(n \log n)$. Klein's result subsumes many of the previous results and goes far beyond them. There are, however, situations, e.g., circle sites under Euclidean distance, where it is not known how to determine $L$ and $R$ in the divide-andconquer algorithm such that their bisector is acyclic; see [23].

The purpose of this paper is to show that there is an $O(n \log n)$ randomized algorithm for constructing (a subset of Klein's) abstract Voronoi diagrams even without the acyclicity assumption. The subset is defined by the following two general position assumptions: we do not allow bisecting curves to touch but require that all intersections are crossings and that no four bisecting curves go through a common point.

The algorithm is given in Section 3 and applications can be found in Section 4. In many concrete situations, e.g., point sites with Euclidean distance function, our algorithm is just another $O(n \log n)$ algorithm, albeit simpler. There are however at least two cases where we achieve $O(n \log n)$ for the first time: For disjoint convex sites the best deterministic algorithm runs in time $O\left(n(\log n)^{2}\right)$ [19] and for line segments under the Haussdorff metric, i.e., a point $x$ and a line segment $s=\overline{s_{1} s_{2}}$ have distance $\max \left(\left|x-s_{1}\right|,\left|x-s_{2}\right|\right)$, an $O(n \log n)$ algorithm was only known in the special case of so-called $\alpha$-disjoint segments [3]. We also want to stress that the new algorithm is uniform in the sense that only a small number of primitives, see Section 2, are problem specific.

Our algorithm is based on Clarkson and Shor's randomized incremental construction technique [7]. The idea is to construct the abstract Voronoi diagram of a set $S$ of sites incrementally by adding site after site in random order. When $R \subseteq S$ is the current set of sites, the Voronoi diagram $V(R)$ and a conflict graph $G(R)$ is maintained. The conflict graph contains all pairs $\{e, t\}$, where $e$ is an edge of $V(R)$ and $t \in S-R$ is a site still to be considered, such that addition of site $t$ causes the edge $e$ to be removed (either completely or partially) from the diagram. In order to make Clarkson and Shor's method applicable we have to show that for a site $s \in S-R$ the diagram $V(R \cup\{s\})$ and the conflict graph $G(R \cup\{s\})$ can be constructed from $V(R)$ and $G(R)$ in time

$$
O\left(\sum_{\{e, s\} \in G(R)} \operatorname{deg}_{G(R)}(e)\right)
$$


where $\operatorname{deg}_{G(R)}(e)$ is the degree of $e$ in $G(R)$ and the summation is over all edges $e$ of $V(R)$ which conflict with new site $s$. This is the content of Theorem 1 of Section 3. If the method is applicable the expected running time is

$$
O\left(n+m(n)+n \cdot \sum_{1 \leq r \leq n / 2} m(r) / r^{2}\right)
$$

where $m(r)$ is the expected number of edges in $V(R)$. For abstract diagrams $m(r) \leq 3 r$ and hence the algorithm runs in time $O(n \log n)$.

Throughout we use the following notation: For a subset $X \subseteq \mathbb{R}^{2}$ the closure, boundary, and interior of $X$ are denoted by $\mathrm{cl} X$, bd $X$, and int $X$, respectively.

\section{Abstract Voronoi Diagrams}

Let $n \in \mathbb{N}$, and for each pair of integers $p, q$ such that $1 \leq p \neq q<n$ let $D(p, q)$ be either empty or an open unbounded subset of $\mathbb{R}^{2}$ and let $J(p, q)$ be the boundary of $D(p, q)$. We postulate:

(1) $J(p, q)=J(q, p)$ and for each $p, q$ such that $p \neq q$ the regions $D(p, q), J(p, q)$, and $D(q, p)$ form a partition of $\mathbb{R}^{2}$ into three disjoint sets.

(2) If $\varnothing \neq D(p, q) \neq \mathbb{R}^{2}$, then $J(p, q)$ is homeomorphic to the open interval $(0,1)$.

We call $J(p, q)$ the bisecting curve for sites $p$ and $q$. The abstract Voronoi diagram is now defined as follows:

\section{Definition [13].}

(a) Let $S=\{1, \ldots, n-1\}$ and

$$
\begin{aligned}
R(p, q) & := \begin{cases}D(p, q) \cup J(p, q) & \text { if } p<q, \\
D(p, q) & \text { if } p>q,\end{cases} \\
\operatorname{VR}(p, S) & :=\bigcap_{\substack{q \in S \\
q \neq p}} R(p, q), \\
V(S) & :=\bigcup_{p \in S} \operatorname{bd} \operatorname{VR}(p, S) .
\end{aligned}
$$

$\operatorname{VR}(p, S)$ is called the Voronoi region of $p$ with respect to $S$ and $V(S)$ is called the Voronoi diagram of $S$.

(b) We postulate that the Voronoi regions and the bisecting curves satisfy the following two conditions:

(1) Any two bisecting curves have only a finite number of points in common. Any point in common to two bisecting curves is a proper crossing between the two curves, see Fig. 1. 

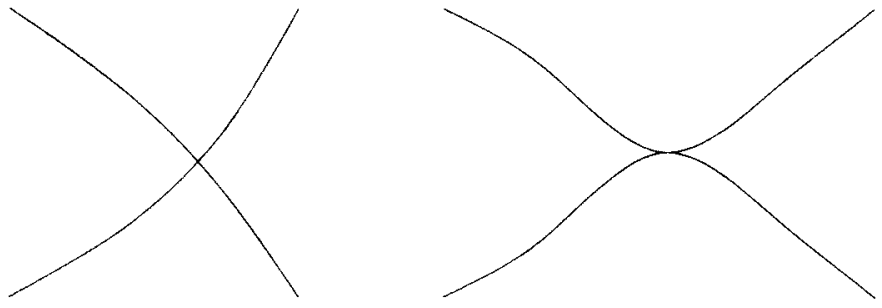

Fig. 1. A crossing and touching point.

(2) For any nonempty subset $S^{\prime}$ of $S$

(A) if $\operatorname{VR}\left(p, S^{\prime}\right)$ is nonempty, then $\operatorname{VR}\left(p, S^{\prime}\right)$ is path-connected and has nonempty interior for each $p \in S^{\prime}$

(B) $\mathbb{R}^{2}=\bigcup_{p \in S^{\prime}} \operatorname{VR}\left(p, S^{\prime}\right)$ (disjoint)

Remark. Klein's definition is actually more liberal. He allows that bisecting curves may touch and only requires that their intersection consists of finitely many connected components. In $(2 \mathrm{~A})$ he postulates that each $\operatorname{VR}\left(p, S^{\prime}\right)$ is non empty. The weaker assumption made here does not harm his theory.

Fact 1 [15].

(a) Voronoi regions are simply connected.

(b) The following holds for each point $v \in V(S)$ : There are arbitrarily small neighborhoods $U$ of $v$ that have the following properties. Let $\operatorname{VR}\left(p_{1}, S\right)$, $\operatorname{VR}\left(p_{2}, S\right), \ldots, \operatorname{VR}\left(p_{k}, S\right)$ be the sequence of Voronoi regions traversed on a counterclockwise march around the boundary of $U$ and let $I_{1}, I_{2}, \ldots, I_{k}$ denote the corresponding intervals of bd $U$, where $I_{j}=\left\langle w_{j}, w_{j+1}\right\rangle \subseteq$ $\operatorname{VR}\left(p_{j}, S\right)$ for $1 \leq j \leq k$ (indices must be read $\bmod k$ ). The intervals may be open, half-open, or closed. We have $w_{j} \neq w_{j+1}$ for $1 \leq j \leq k$. The common boundary of $\operatorname{VR}\left(p_{j-1}, S\right)$ and $\operatorname{VR}\left(p_{j}, S\right)$ defines a curve segment $\beta_{j} \subseteq$ $J\left(p_{j-1}, p_{j}\right)$ connecting $v$ and $w_{j} . V(S) \cap U$ is the union of the curve segments $\beta_{j}$ together with the point $v$. Each $\beta_{j}$ is contained in the Voronoi region of $\min \left\{p_{j-1}, p_{j}\right\}$. The open "piece of pie" bordered by $\beta_{j}, \beta_{j+1}$, and $I_{j}$ belongs to $\operatorname{VR}\left(p_{j}, S\right)$. The point $v$ belongs to the region of $\min \left\{p_{1}, \ldots, p_{k}\right\}$. Finally, $p_{i} \neq p_{j}$ for $i \neq j$.

For the following it is helpful to restrict attention to the "finite part" of $V(S)$. Let $\Gamma$ be a simple closed curve such that all intersections between bisecting curves lie in the inner domain of $\Gamma$. We add a site $\infty$ to $S$, define $J(p, \infty)=J(\infty, p)=\Gamma$ for all $p, 1 \leq p<n$, and $D(\infty, p)$ to be the outer domain of $\Gamma$ for each $p, 1 \leq p<n$.

Fact 2 [15]. The boundary of each nonempty Voronoi region is a simple closed curve. Moreover, the closure of each nonempty Voronoi region $\operatorname{VR}(p, S), p \neq \infty$, is homomorphic to a closed disc. A Voronoi diagram can be represented as a planar graph in a natural way. The vertices of the graph are the points of $V(S)$ which 
belong to the boundary of three or more Voronoi regions and the edges of the graph correspond to the maximal connected subsets of $V(S)$ belonging to the boundary of exactly two Voronoi regions. The faces of the graph correspond to the nonempty Voronoi regions. We use $V(S)$ to denote this graph also. For the algorithmic treatment of Voronoi diagrams we also need to make a feasibility assumption about the bisecting curves.

Definition [15]. The following operations on bisecting curves are assumed to take time $O(1)$.

(1) Given $J(p, q)$ and a point $v$, determine if $v \in D(p, q)$ holds.

(2) Given a point $v$ in common to three bisecting curves, determine the clockwise order of the curves around $v$.

(3) Given points $v \in J(p, q)$ and $w \in J(p, r)$ and orientations of these curves, determine the first point of $\left.J(p, r)\right|_{(w, \infty)}$ crossed by $\left.J(p, q)\right|_{(v, \infty)}$.

(4) Given $J(p, q)$ with an orientation, and points $v, w, x$ on $J(p, q)$, determine if $v$ comes before $w$ on $\left.J(p, q)\right|_{(x, \infty)}$.

For simplicity we also make the following general position assumption.

General Position Assumption: No four bisecting curves have a point in common.

The general position assumption and Fact 1 imply that each vertex of the Voronoi diagram has degree three. It lies at the intersection of three bisecting curves as shown in Fig. 2.

Remark. The requirement that the Voronoi regions partition the plane is a severe restriction on the family of bisecting curves. Consider a crossing of $J(r, p)$ and $J(r, q)$ as in Fig. 3. Then $J(p, q)$ must also pass through $v$ with $D(q, p)$ on its right.

We close this section with a simple but important property of Voronoi edges:

Lemma 1. Let $R \subseteq S$ and $t \in S-R$. Let $e$ be an edge of $V(R)$ which separates the regions $\operatorname{VR}(p, R)$ and $\operatorname{VR}(q, R)$ of the two sites $p, q \in R$. Then $e \cap \operatorname{VR}(t, R \cup\{t\})=$ $e \cap \operatorname{VR}(t,\{p, q, t\})$.

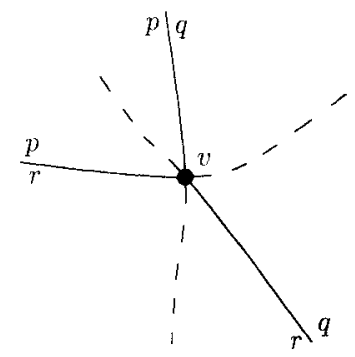

Fig. 2. The bisecting curves $J(p, q), J(p, r), J(r, q)$ intersect at $v$. The domains $D(p, q)$ and $D(q, p)$ are indicated by the letters $p$ and $q$ on the two sides of the bisecting curve $J(p, q)$. The parts of the bisecting curves which define region boundaries are shown as solid lines. 


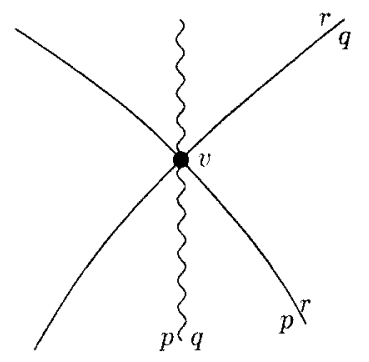

Fig. 3.

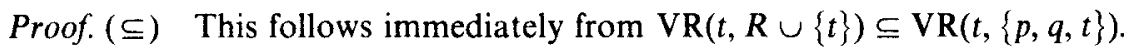

(巳) Let $x \in e \cap \operatorname{VR}(t,\{p, q, t\})$. From $x \in e$ we conclude $x \in \operatorname{VR}(p, R) \cup$ $\operatorname{VR}(q, R)$ and hence $x \notin \operatorname{VR}(r, R) \supseteq \operatorname{VR}(r, R \cup\{t\})$ for any $r \in R-\{p, q\}$. From $x \in \operatorname{VR}(t,\{p, q, t\})$ we conclude $x \notin \operatorname{VR}(p,\{p, q, t\}) \cup \operatorname{VR}(q,\{p, q, t\}) \supseteq$ $\operatorname{VR}(p, R \cup\{t\}) \cup \operatorname{VR}(q, R \cup\{t\})$. Thus $x \notin \operatorname{VR}(r, R \cup\{t\})$ for any $r \in R$ and hence $x \in \operatorname{VR}(t, R \cup\{t\})$.

Informally, Lemma 1 states that the influence of a site on a given edge depends only on the sites defining this particular edge.

\section{Incremental Construction of Abstract Voronoi Diagrams}

In this section we describe the incremental construction algorithm. We start with three sites $\infty, p, q$ where $p$ and $q$ are chosen at random and then add the remaining sites in random order. At the general step we have to consider a set $R \subseteq S$ of sites with $\infty \in R$ and $|R| \geq 3$. We maintain the following data structures.

(1) The Voronoi diagram $V(R)$ : It is stored as a planar graph as described in the previous section.

(2) The conflict graph $G(R)$ : The vertices of the conflict graph $G(R)$ are the edges of $V(R)$ and the sites in $S-R$. There is an edge (read: conflict) between the edge $e$ of $V(R)$ and the site $s \in S-R$ iff $e \cap \operatorname{VR}(s, R \cup\{s\}) \neq$ $\varnothing$.

Remark. Recall that an edge of a Voronoi diagram is an open set and that a Voronoi region may contain part of its boundary. For the definition of conflict graph it is however immaterial whether we intersect open sets or their closures.

Lemma 2. $\operatorname{cl} e \cap \mathrm{cl} \mathrm{VR}(s, R \cup\{s\}) \neq \varnothing$ implies en $\operatorname{VR}(s, R \cup\{s\}) \neq \varnothing$

Proof. Let $x \in \mathrm{cl} e \cap \mathrm{cl} \operatorname{VR}(s, R \cup\{s\})$. Assume first that $x$ is an endpoint of $e$. Then $x$ lies at the intersection of three bisecting curves of sites in $R$. Hence no bisecting curve $J(s, r), r \in R$, can go through $x$ and therefore an entire neighborhood of $x$ must belong to $\operatorname{VR}(s, R \cup\{s\})$. Thus $e \cap \operatorname{VR}(s, R \cup\{s\}) \neq \varnothing$. Assume 
next that $x \in e \cap \operatorname{bd} \operatorname{VR}(s, R \cup\{s\})$. Then $x \in J(p, q) \cap J(s, r)$ for some sites $p, q, r \in R$. The bisecting curves $J(p, q)$ and $J(s, r)$ cross at point $x$ and hence there is a point $y \in e$ in the neighborhood of $x$ such that $y \in \operatorname{VR}(s, R \cup\{s\})$.

We next discuss how to update the data structures after the addition of a site $s \in S-R$ to $R$. We first concentrate on the construction of the Voronoi diagram $V(R \cup\{s\})$ from $V(R)$ and $G(R)$.

Let $\mathscr{S}=\operatorname{VR}(s, R \cup\{s\})$. We proceed in several steps. Lemma 3 deals with the case $\mathscr{S}=\varnothing$. The case $\mathscr{S} \neq \varnothing$ is dealt with in Lemmas 4 and 5 . We show that the intersection of the current diagram $V(R)$ with the region $\mathscr{S}$ is a connected set (Lemma 4) and that the intersection $e \cap \mathscr{S}$ for an edge $e$ of $V(R)$ consists of at most two components (Lemma 5). From Lemmas 4 and 5 we derive the update algorithm.

Lemma 3. $\mathscr{S}=\varnothing$ iff $\operatorname{deg}_{G(R)}(s)=0$.

Proof. If $\mathscr{S}=\varnothing$, then clearly $\operatorname{deg}_{G(R)}(s)=0$. So let us assume $\mathscr{S} \neq \varnothing$. If $\operatorname{deg}_{G(R)}(s)=0$, then $\operatorname{cl} \mathscr{S} \subseteq$ int $\operatorname{VR}(r, R)$ for some $r \in R$. Next observe that $\operatorname{VR}(r, R \cup\{s\})=\operatorname{VR}(r, R)-\mathscr{S}$. Also $r \neq \infty$ since $\operatorname{VR}(\infty, P)$ is the outer domain of the closed curve $\Gamma$ for all $P, \infty \in P \subseteq S$. Thus $\operatorname{VR}(r, R \cup\{s\})$ is bounded but not simply connected. This contradicts Fact 1(a).

If $\mathscr{S}=\varnothing$, then $V(R \cup\{s\})=V(R)$. So let us assume $\mathscr{S} \neq \varnothing$ and hence $\operatorname{deg}_{G(R)}(s) \neq 0$. Let $I=V(R) \cap \operatorname{cl} \mathscr{P}$.

Lemma 4. I is a connected set which intersects bd $\mathscr{P}$ in at least two points.

Proof. The boundary bd $\mathscr{S}$ is a simple closed curve which does not go through any vertex of $V(R)$. This follows from Fact 2 and the general position assumption. Also $I \neq \varnothing$ by Lemma 2. Let $I_{1}, I_{2}, \ldots, I_{k}$ be the connected components of $I$.

Claim. Each $I_{j}, 1 \leq j \leq k$, contains two points of bd $\mathscr{T}$.

Proof. Assume first that $I_{j}$ contains no points of bd $\mathscr{S}$, i.e., $I_{j} \subseteq$ int $\mathscr{S}$. Then there is a simple closed curve $C \subset$ int $\mathscr{S}$ such that $I_{j}$ is contained in the inner domain of $C$ and $C$ does not intersect $V(R)$. Thus $C \subseteq$ int $\operatorname{VR}(r, R)$ for some $r \in R$. Since Voronoi regions are simply connected, $C$ and its interior must belong to $\operatorname{VR}(r, R)$ and hence $C$ cannot contain a component $I_{j}$ in its interior. Assume next that $I_{j}$ intersects bd $\mathscr{P}$ in exactly one point, say $x$. Then there is a simple closed curve $C$ containing $I_{j}$ in its inner domain such that $x \in C, C-\{x\} \subseteq$ int $\mathscr{P}$ and $C-\{x\}$ does not intersect $V(R)$. Thus $C-\{x\} \subseteq \mathrm{VR}(r, R)$ for some $r \in R$ and hence $x$ is a point on an edge of $V(R)$ such that both sides of the edge belong to the same Voronoi region. This contradicts Fact 1.

Assume now that $k \geq 2$. Then there is a path $P \subseteq \mathrm{cl} \mathscr{S}-\left(I_{1} \cup \cdots \cup I_{k}\right)$ connecting two points on the boundary bd $S$ such that one component of $\mathscr{S}-P$ 


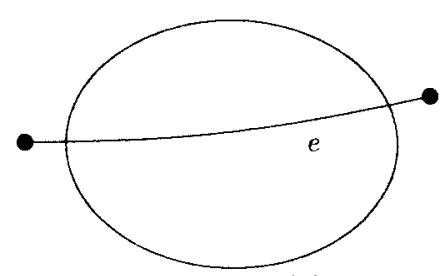

bd $\mathscr{S}$

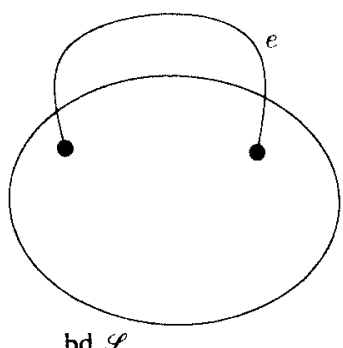

bd $\mathscr{S}$

Fig. 4. Two possible cases of Lemma 5.

contains $I_{1}$ and the other component contains $I_{2}$. Let $x$ and $y$ be the endpoints of $P$ and let $r \in R$ be such that $P \subseteq \mathrm{VR}(r, R)$. Since $x, y \notin V(R)$ we conclude that $V R(r, R \cup\{s\})=\operatorname{VR}(r, R)-\mathscr{P} \neq \varnothing$. Thus $x, y \in \operatorname{cl} \operatorname{VR}(r, R \cup\{s\})$ and hence there is a simple path $Q \subseteq \operatorname{cl} \operatorname{VR}(r, R \cup\{s\})$ with endpoints $x$ and $y$. The cycle $P \circ Q$ is then contained in $\operatorname{cl} \operatorname{VR}(r, R)$ and contains either $I_{1}$ or $I_{2}$ in its interior. Thus $\operatorname{VR}(r, R)$ is not simply connected, a contradiction to Fact 2.

Lemma 5. Let $e$ be an edge of $V(R)$. If $e \cap \mathscr{S} \neq \varnothing$, then either $e \cap \mathscr{S}=V(R) \cap \mathscr{S}$ and $e \cap \mathscr{S}$ is a single component or $e-\mathscr{S}$ is a single component; see Fig. 4.

Proof. Assume first that $e \cap \mathscr{S}=V(R) \cap \mathscr{S}$. Since $V(R) \cap \mathscr{S}$ is connected by Lemma 4 we conclude that $e \cap \mathscr{S}$ is connected. Assume next that $e \cap \mathscr{S} \neq$ $V(R) \cap \mathscr{S}$. Then with every point $x \in e \cap \mathscr{S}$ one of the subpaths of $e$ connecting $x$ to an endpoint of $e$ must be contained in $\mathscr{S}$. Hence $e-\mathscr{S}$ is a single component.

Let $L=\{e$ edge of $V(R) ;\{e, s\} \in G(R)\}$. For $e \in L$ let $e^{\prime}=e \cap \mathscr{P}$. Note that $e^{\prime}=e \cap \operatorname{VR}(s,\{p, q, s\})$ by Lemma 1 where $e$ separates the regions of sites $p$ and $q$; hence $e^{\prime}$ can be computed from $e$ in time $O(1)$. We have shown above that the set $\bigcup_{e \in L} \mathrm{cl} e^{\prime}=V(R) \cap \mathrm{cl} \mathscr{S}$ is connected. Let $B=\left\{x ; x\right.$ is an endpoint of $e^{\prime}$ which is not an endpoint of $e$ for some $e \in L\}=V(R) \cap$ bd $\mathscr{S}$. Since bd $\mathscr{S}$ is a simple closed curve by Fact 2 , bd $\mathscr{S}$ induces a cyclic ordering on the points in $B$. Since $V(R) \cap \mathrm{cl} \mathscr{S}$ is connected this cyclic ordering can be determined by a traversal of the planar graph $V(R) \cap \mathrm{cl} \mathscr{S}$. It is now easy to update the Voronoi diagrams as follows:

Step 1. Compute $e^{\prime}$ for each $e \in L$. Remove $e^{\prime}$ from $V(R)$ for each $e \in L$.

Step 2. Compute $B$ and the cyclic ordering on $B$ induced by bd $\mathscr{S}$.

Step 3. Let $x_{1}, \ldots, x_{k}$ be the set $B$ in its cyclic ordering and let $r_{i} \in R$ be such that $\left\{x_{\mathrm{i}}, x_{i+1}\right\} \subseteq \operatorname{bd} \operatorname{VR}\left(r_{i}, R\right)$.

(1) for $i$ from 1 to $k$

(2) do add the part of $J\left(r_{i}, s\right)$ with endpoints $x_{i}$ and $x_{i+1}$ to the Voronoi diagram

(3) od 
For the time bound we only have to observe that steps 1 and 2 take time $O(|L|)$ and that step 3 takes time $O(k)=O(|L|)$. This proves the following

Lemma 6. Let $s \in S-R$. Then $V(R \cup\{s\})$ can be constructed from $V(R)$ and $G(R)$ in time $O\left(\operatorname{deg}_{G(R)}(s)+1\right)$.

We now turn to the update of the conflict graph.

Lemma 7. Let $s \in S-R$. Then $G(R \cup\{s\})$ can be constructed from $V(R)$ and $G(R)$ in time

$$
O\left(\sum_{\{e, s\} \in G(R)} \operatorname{deg}_{G(R)}(e)\right)
$$

Proof. In this proof we distinguish three cases: edges of $V(R \cap\{s\})$ which were already edges of $V(R)$, edges which are part of edges of $V(R)$, and edges which are completely new. The only difficult case is the third one; it is dealt with in Lemma 8.

As above let $L=\{e ; e$ is an edge of $V(R)$ and $e \cap \mathscr{S} \neq \varnothing\}$ where $\mathscr{S}=$ $\operatorname{VR}(s, R \cup\{s\})$. For $e \notin L$ the conflict information does not change. This follows from

$$
e \cap \operatorname{VR}(t, R \cup\{s, t\})=e \cap(\operatorname{VR}(t, R \cup\{t\})-\operatorname{VR}(s, R \cup\{s, t\}))
$$

and

$$
(e \cap \operatorname{VR}(t, R \cup\{t\}))-(e \cap \operatorname{VR}(s, R \cup\{s, t\}))=e \cap \operatorname{VR}(t, R \cup\{t\})
$$

Let us next consider an edge $e \in L$. If $e \subseteq \mathscr{S}$, then $e$ has to be deleted from the conflict graph. This certainly takes time $O\left(\operatorname{deg}_{G(R)}(e)\right)$. If $e \nsubseteq \mathscr{S}$, then $e-\mathscr{S}$ consists of at most two subsegments by Lemma 5. Let $e^{\prime}$ be one of those subsegments and let $t \in S-R-\{s\}$. Then $e^{\prime} \cap \operatorname{VR}(t, R \cup\{s\} \cup\{t\})=$ $e^{\prime} \cap \bigcap_{r \in R} R(t, r) \cap R(t, s)=e^{\prime} \cap \operatorname{VR}(t, R \cup\{t\}) \cap R(t, s) \subseteq e \cap \operatorname{VR}(t, R \cup\{t\})$ and hence any site $t$ in conflict with $e^{\prime}$ must be in conflict with $e$.

It remains to consider those edges of $V(R \cup\{s\})$ which are not fragments of edges of $V(R)$. Let $e_{12}$ be one of those edges. The endpoints $x_{1}$ and $x_{2}$ of $e_{12}$ lie in the interior of edges $e_{1}$ and $e_{2}$ on $\operatorname{bd} \operatorname{VR}(p, R)$ for some $p \in R$. Also $e_{12}$ is part of the bisecting curve $J(p, s)$. Note that $p \neq \infty$ since $J(\infty, s)=\Gamma \subseteq V(R)$. Let $P$ be that part of $\operatorname{bd} \operatorname{VR}(p, R)$ which connects $x_{1}$ and $x_{2}$ and is contained in $\mathscr{S}$ in all sufficiently small neighborhoods of $x_{1}$ and $x_{2}$.

Claim. $P \subseteq \mathscr{P}$

Proof. bd VR $(p, R)$ is a simple closed curve and int $\operatorname{VR}(p, R)$ is the bounded domain defined by this curve. Assume now that $P$ crosses bd $\mathscr{P}$. Then $\operatorname{VR}(p, R \cup\{s\})=\operatorname{VR}(p, R)-\mathscr{S}$ is not connected, a contradiction.

Lemma 8. Let $t \in S-R-\{s\}$, and let $t$ conflict with $e_{12}$ in $V(R \cup\{s\})$. Then $t$ conflicts in $V(R)$ with either $e_{1}$ or $e_{2}$ or one of the edges of $P$. 


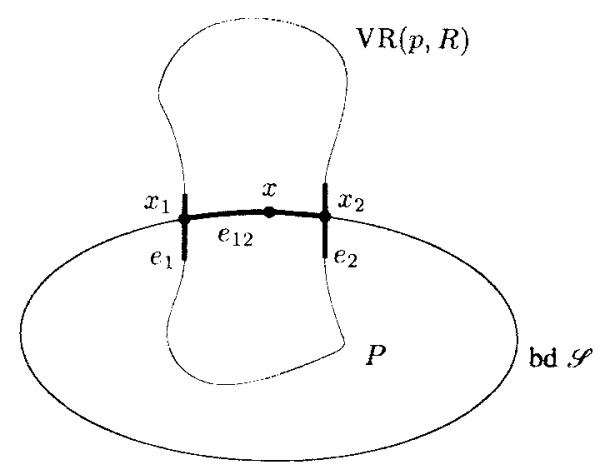

Fig. 5.

Proof. Consider $\operatorname{VR}(p, R)$ (Fig. 5). By the definition of conflict a point $x \in e_{12}$ exists such that $x \in \mathrm{VR}(t, R \cup\{s, t\}) \subseteq \mathrm{VR}(t, R \cup\{t\})$. Since we claim a contradiction we assume that $t$ is not in conflict with $P, e_{1}$, or $e_{2}$ in $V(R)$. Thus, $\mathrm{VR}(t, R \cup\{s, t\}) \cap U\left(x_{1}\right) \subseteq \operatorname{VR}(t, R \cup\{t\}) \cap U\left(x_{1}\right)=\varnothing$ for any sufficiently small neighborhood $U\left(x_{1}\right)$ of $x_{1}$. Now consider in any such neighborhood of $x_{1}$ the wedge spanned by $e_{12}$ and the part of $e_{1}$ outside $\mathscr{S}$. The points in this wedge all belong to $\operatorname{VR}(p, R \cup\{s, t\})$. The same is true for any sufficiently small neighborhood of $x_{2}$ with $e_{2}$ instead of $e_{1}$. Since $\operatorname{VR}(p, R \cup\{s, t\})$ is connected, there is a path $Q$ from $x_{1}$ to $x_{2}$ running completely inside $\operatorname{VR}(p, R \cup\{s, t\}) \subseteq \operatorname{VR}(p, R \cup\{t\})$ except at the endpoints (see Fig. 6). By definition of $P$ and $Q$ the Voronoi region $\operatorname{VR}(t, R \cup\{t\})$ cannot intersect these two paths. Moreover, $x$ lies in the interior of the cycle $x_{1} \circ P \circ x_{2} \circ Q$; otherwise $\operatorname{VR}(p, R)$ would not be simply connected. From $x_{1}, x_{2} \notin \operatorname{VR}(t, R \cup\{t\})$ and $x \in \operatorname{VR}(t, R \cup\{t\})$ we conclude that $\operatorname{VR}(t, R \cup\{t\})$ lies in the interior of the cycle. This is a contradiction to the fact that $\operatorname{VR}(p, R \cup\{t\})$ is simply connected.

Lemmas 8 and 1 together allow us to compute the conflict information for the new edges. Let $e_{12} \subseteq J(p, s)$ be any new edge. A site $t$ in conflict with edge $e_{12}$ must have conflicted in $G(R)$ with either $e_{1}, e_{2}$ or one of the edges on the path $P$ by Lemma 8. Also for any such site $t$ we can compute the conflict information $e_{12} \cap \operatorname{VR}(t, R \cup\{s, t\})$ in time $O(1)$ by Lemma 1. Thus the set of neighbors of edge

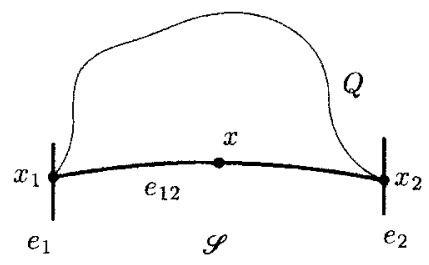

Fig. 6. 
$e_{12}$ in $G(R \cup\{s\})$ can be computed in time

$$
O\left(\sum_{e \in P \cup\left\{e_{1}, e_{2}\right\}} \operatorname{deg}_{G(R)}(e)\right),
$$

where the sum is over all edges in $P \cup\left\{e_{1}, e_{2}\right\}$. Next observe that every edge $e \in V(R)$ with $e \cap \operatorname{VR}(s, R \cup\{s\}) \neq \varnothing$ can belong at most twice to a path $P$ for some new edge by planarity. Thus $G(R \cup\{s\})$ can be obtained from $G(R)$ in time

$$
O\left(\sum_{\{e, s\} \in G(R)} \operatorname{deg}_{G(R)}(e)\right)
$$

This proves Lemma 7 .

\section{Theorem 1.}

(a) Let $s \in S-R$. Then the data structures $G(R \cup\{s\})$ and $V(R \cup\{s\})$ can be obtained from $G(R)$ and $V(R)$ in time

$$
O\left(\sum_{\{e, s\} \in G(R)} \operatorname{deg}_{G(R)}(e)\right) .
$$

(b) For $R \subseteq S,|R|=3$, and $\infty \in R$ the data structures $V(R)$ and $G(R)$ can be set up in time $O(n)$ where $n=|S|$.

Proof. (a) This point summarizes Lemmas 6 and 7.

(b) The Voronoi diagram $V(R)$ for three sites $\infty, p$, and $q$ has the structure shown in Fig. 7 and can certainly be set up in time $O(1)$. Also for each of the edges $e$ of $V(R)$ and each of the $n-3$ sites in $S-R$ we can test $e \cap \operatorname{VR}(t, R \cup\{t\}) \neq \varnothing$ in $O(1)$ by Lemma 1 . This proves (b).

Lemma 9. The number of edges of $V(R)$ is at most $3|R|$.

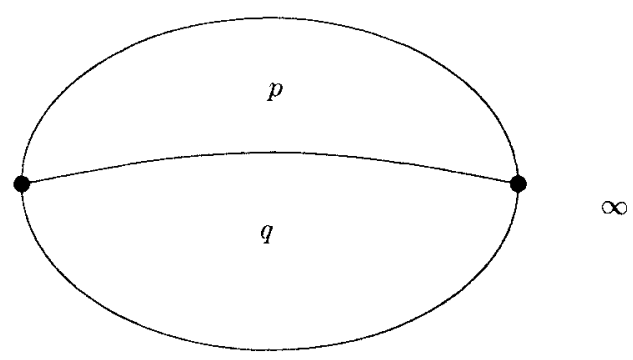

Fig. 7. The Voronoi diagram for sites $\infty, p$, and $q$. 
Proof. $V(R)$ is a planar graph with at most $|R|$ regions. Also, each vertex has degree three. The number of edges is therefore at most $3|R|$ by Euler's formula.

Theorem 2. The abstract Voronoi diagram $V(S)$ of $n$ sites can be constructed by a randomized algorithm in time $O(n \log n)$.

Proof. In [7] Clarkson and Shor show that randomized incremental construction has expected running time

$$
O\left(m(n)+n \cdot \sum_{1 \leq r \leq n / 2} m(r) / r^{2}+n\right)
$$

provided that initialization takes time $O(n)$ and addition of an object (here site) $s$ to the set $R$ takes time proportional to

$$
\sum_{\{e, s\} \in G(R)} \operatorname{deg}_{G(R)}(e)
$$

where the summation is over all regions (here edges) of the current structure (here Voronoi diagram $V(R)$ ) which conflict with site $s$. Also $m(r)$ is the expected size of the structure for a random subset $R \subseteq S$ of $r$ elements. In our case we have $m(r) \leq 3 r$ by Lemma 9 . Finally, the assumptions of Clarkson's theorem are satisfied by Theorem 1 . The time bound follows.

Remark. In our alogorithm $\infty \in R$ always. An inspection of Clarkson's argument shows that this minor deviation from randomness does not change the time bound.

\section{Applications}

Many previously considered types of Voronoi diagrams fall under the framework described above.

1. Point Sites. In their pioneering paper Shamos and Hoey [22] showed how to construct the Voronoi diagram for point sites under the Euclidean metric in time $O(n \log n)$. This was later extended to arbitrary $L_{p}$-metrics, $1 \leq p \leq \infty$, by Lee [18], to the $L_{2}$-metric with additive weights by Sharir [23] and Fortune [10], to the so-called Moscow-metric by Klein [14], to convex distance functions by Chew and Drysdale [6] and Fortune [9], and to abstract Voronoi diagrams by Klein and Wood [17] and Klein [13]. The previous algorithms for abstract diagrams had to assume, as they were based on the divide-and-conquer approach, that the set of sites $S$ can be partitioned into about equal-sized subsets $L$ and $R$ such that the bisector between $L$ and $R$ is acyclic. This assumption is crucial for the efficiency of the merging step. For all cases mentioned our algorithm gives an alternative 


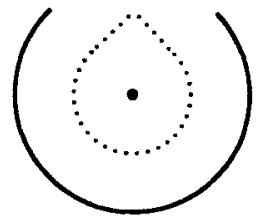

Fig. 8. The bisector for a point and a circular arc.

$O(n \log n)$ solution, albeit randomized. For abstract diagrams [13] we do not need the acyclicity assumption, however, and for the $L_{p}$-norm we may also add additive weights.

2. Beyond Point Sites. Point and line sites were considered by Kirkpatrick [12] and Fortune [10], and disjoint convex objects were considered by Leven and Sharir [19]. In the latter case, the running time is $O\left(n(\log n)^{2}\right)$ since the Leven-Sharir algorithm uses divide-and-conquer and the bisector between the subsets $L$ and $R$ of $S$ mentioned above is not necessarily acyclic. Our algorithm runs in time $O(n \log n)$. Other applications are the Voronoi diagrams for circles under the Laguerre distance [11], [1], [2] and for disjoint convex polygons under a convex distance function [20].

Of course, there are also types of Voronoi diagrams which do not fall under the framework, e.g., the diagram for points under the Euclidean metric with multiplicative weight [4], the diagram for points and circular arcs, and the diagram for points under metrics which arise from weighted partitions of the plane [21]. In all three cases the bisector $J(p, q)$ of two sites may be a closed curve, see Fig. 8.

\section{Conclusions and Open Problems}

We showed that Clarkson and Shor's randomized incremental construction method works for (a subset of) Klein's abstract Voronoi diagrams. Many previously considered types of Voronoi diagrams can thus be handled by the same simple algorithm. In [16] the results of this paper are extended in two ways. We show that the algorithm can be programmed on a schema level such that specific Voronoi diagram algorithms can be derived in a simple way; we also drop the general position assumption and the assumption that bisecting curves may not touch. Nevertheless, many open problems remain:

(1) Can the concept of abstract Voronoi diagram be generalized to higher dimensions?

(2) What can be done in two dimensions without the assumption that bisectors are nonclosed curves?

(3) Can the algorithm be modified in order to handle higher-order Voronoi diagrams? 


\section{References}

1. Aurenhammer, F., Power diagrams: properties, algorithms and applications, SIAM J. Comput. 16 (1987), 78-96.

2. Aurenhammer, F., Improved algorithms for discs and balls using power diagrams, J. Algorithms 9 (1988), $151-161$.

3. Aurenhammer, F., Voronoi Diagrams - A Survey, Technical Report 263, Institutes for Information Processing, Graz Technical University (1988).

4. Aurenhammer, F., and Edelsbrunner, H., An optimal algorithm for constructing the weighted Voronoi diagram in the plane, Pattern Recognition 17 (2) (1984), 251 - 257.

5. Brown, K. Q., Voronoi diagrams from convex hulls, IPL 9 (1979), 223-228

6. Chew, L. P., and Drysdale III, R. L., Voronoi diagrams based on convex distance functions, Proc. Ist ACM Symp. on Computational Geometry (1985), pp. 235-244.

7. Clarkson, K. L., and Shor, P. W., Applications of random sampling in computational geometry, II, Discrete Comput. Geom. 4 (1989), 387-421.

8. Edelsbrunner, H. and Seidel, R., Voronoi diagrams and arrangements, Discrete Comput. Geom. 1 (1986), $25-44$.

9. Fortune, S., A fast algorithm for polygon containment by translation (extended abstract), Proc. 12th Int. Colloq. on Automata, Languages, and Programming (1985), Lecture Notes in Computer Science, Vol. 194, Springer-Verlag, Berlin, pp. 189-198.

10. Fortune, S., A sweepline algorithm for Voronoi diagrams, Algorithmica 2 (1987), 153-174.

11. Imai, H., Iri, M., and Murota, K., Voronoi diagram in the Laguerre geometry and its applications, SIAM J. Comput. 14 (1985), 93-105.

12. Kirkpatrik, D. G., Efficient computation of continuous skeletons, Proc. 20th IEEE Symp. on Foundations of Computer Science (1979), pp. 18-27.

13. Klein, R., Abstract Voronoi diagrams and their applications (extended abstract), in: H. Noltemeier (ed.), Proc. Computational Geometry and its Applications (CG'88), Würzburg (1988), Lecture Notes in Computer Science, Vol. 333, Springer-Verlag, Berlin, pp. 148-157.

14. Klein, R., Voronoi diagrams in the Moscow metric (extended abstract), in: J. van Leeuwen (ed.), Proc. Graphtheoretic Concepts in Computer Science (WG'88), Amsterdam (1988), Lecture Notes in Computer Science, Vol. 344, Springer-Verlag, Berlin, pp. 434-441.

15. Klein, R., Concrete and Abstract Voronoi Diagrams, Lecture Notes in Computer Science, Vol. 400 , Springer-Verlag, Berlin.

16. Klein, R., Mehlhorn, K., and Meiser, S., On the construction of abstract Voronoi diagrams, II, Proc. SIGAL Symp. on Algorithms, Tokyo (1990), Lecture Notes in Computer Science, Vol. 450, SpringerVerlag, Berlin, pp. 138-154.

17. Klein, R., and Wood, D., Voronoi diagrams based on general metrics in the plane, in: R. Cori and M. Wirsing (eds.), Proc. Sth Annual Symp. on Theoretical Aspects of Computer Science (STACS), Bordeaux (1988), Lecture Notes in Computer Science, Vol. 294, Springer-Verlag, Berlin, pp. $281-291$.

18. Lee, D. T., Two-dimensional Voronoi diagrams in the $L_{p}$-metric, J. Assoc. Comput. Mach. 27 (1980), 604-618.

19. Leven, D., and Sharir, M., Intersection and proximity problems and Voronoi diagrams, in: J. Schwartz and C. K. Yap (eds.), Advances in Robotics, Vol. 1 (1986), Erlbaum, Hillsdale, NJ, pp. $187-228$.

20. Leven, D., and Sharir, M., Planning a purely translational motion for a convex object in twodimensional space using generalized Voronoi diagrams, Discrete Comput. Geom. 2 (1987), 9 31.

21. Mitchell, J. S. B., and Papadimitriou, C. H., The weighted region problem, Proc. 3rd ACM Symp. on Computational Geometry, Waterloo (1987), pp. 30-38.

22. Shamos, M. I., and Hoey, D., Closest point problems, Proc. 16th IEEE Symp. on Foundations of Computer Science (1975), pp. 151-162.

23. Sharir, M., Intersection and closest-pair problems for a set of planar discs, SIAM J. Comput. 14 (1985), 448-468.

Received April 28, 1989. 